\title{
Mineração
}

\section{Influência das cavas de extração de areia no balanço hídrico do vale do Paraíba do Sul}

\author{
Benedito Jorge dos Reis \\ Prefeitura Municipal da Estância Turística de Tremembé. E-mail: meioambiente@tremembe.sp.gov.br
}

Getulio Teixeira Batista

Departamento de Ciências Agrárias, UNITAU - Universidade de Taubaté.E-mail: getulio@agro.unitau.br

Marcelo dos Santos Targa

Departamento de Ciências Agrárias, UNITAU - Universidade de Taubaté.E-mail: mtarga@agro.unitau.br

Celso de Souza Catelani

Departamento de Ciências Agrárias, UNITAU - Universidade de Taubaté. E-mail: celso@agro.unitau.br

\section{Resumo}

A crescente formação de lagos artificiais oriundos da atividade de extração de areia no Vale do Rio Paraíba do Sul é responsável por alterações ambientais significativas. Nesse trabalho, é investigada a evolução das áreas de cavas de extração de areia e sua influência no balanço hídrico regional. Para isso, foi desenvolvido um banco de dados georreferenciado, na plataforma SPRING, no qual foram inseridas imagens dos satélites Landsat 5 e 7, cenas 218/76 e 219/76, que foram analisadas para fornecer estimativas das áreas das cavas de areia em um período de 11 anos. Com dados da estação climatológica 83784 - UNITAU/INMET e a área total de lagos artificiais, foi possível estimar o volume de água evaporada para atmosfera a partir dos lagos resultantes dessa atividade. Observou-se um crescimento na área de lagos formados pela extração de areia de 591 ha, em 1993 para 1.726 ha, em 2003. A evaporação resultante aumentou em $203 \%$ e resultou na evaporação de 19.157.022 $\mathrm{m}^{3}$, no ano de 2003, equivalentes ao abastecimento de uma cidade com mais de 326.000 habitantes, considerando a média de consumo do Estado de São Paulo, que é superior à média Brasileira.

Palavras-chave: Extração de areia, balanço hídrico, evaporação de lagos.

\begin{abstract}
The extraction of sand in the valley of the Paraíba do Sul River, carried out in a vulnerable environment, is responsible for significant environmental impacts. This work had the objective of investigating the increase of the total area of lakes created in the mining process for a period of 11 years and of verifying the influence of the evaporation from these lakes on the regional water balance. Thus, a Geographic Information System data basis was implemented using the GIS software package, SPRING, and Landsat imagery. Scenes 218/76 and 219/76 were used for the quantification of the mining areas and for the assessment of the evolution of the activity in the area for a period of 11 years. With data from a local climatological station (UNITAU/INMET-83784) integrated into the database and with the total area of artificial lakes resultant from sand mining activity, it was possible to estimate the increase of the evaporation rate due to this activity. An increase of 591 ha, in 1993 to 1,726 ha in 2003 in the area of lakes was observed. The resulting increase in the evaporating rate was $203 \%$ with 19,157,022 $\mathrm{m}^{3}$ evaporated in 2003 correspondent to the consumption of a city with more than 326,000 inhabitants, considering the average consumption for the State of São Paulo which is greater than the Brazilian average.
\end{abstract}

Keywords: Extraction of sand, water balance, lake evaporation. 


\section{Introdução}

A bacia hidrográfica do rio Paraíba do Sul, situada na Região Sudeste do Brasil, compreendendo parte dos Estados de São Paulo, Rio de Janeiro e Minas Gerais, apresenta grande diversidade e complexidade de uso do solo. A planície aluvial situada no trecho paulista da bacia apresenta uma crescente alteração em seu regime hídrico em razão da evaporação pela formação de lagos resultantes da atividade de extração de areia em cavas. As bacias sedimentares de Taubaté e Rezende contêm grande quantidade de cascalhos e seixos intercalados em leitos de argilas e areias em disposição entrecruzada (São Paulo, 1999). Essa composição e estrutura dos sedimentos funcionam como uma bacia de retenção e liberação de água de infiltração, capaz de manter o rio perene durante todo o ano. O fluxo de base pode chegar à totalidade da vazão do rio durante certas épocas do ano. O rio Paraíba e seus tributários são alimentados continuamente por uma reserva subterrânea, mesmo durante o período de estiagem. Esse fato ocorre devido à existência de camadas de solos permeáveis na planície aluvionar do Paraíba do Sul, principalmente nas formações Caçapava e Resende, e estima-se que, dos $12.795 \mathrm{~km}^{2}$ a montante de Queluz, uma superfície de $8.400 \mathrm{~km}^{2}$ possui características de aquíf́eros (São Paulo, 1977). Esses aqüíferos encontram-se, geralmente, mais próximos da superfície e têm a base de drenagem localizada no leito do rio.

A extração de areia na várzea do rio Paraíba do Sul, para uso na construção civil, corresponde a $10 \%$ da produção nacional (Valverde, 2001) e equivale a $1.023 .836 \mathrm{~m}^{3}$ mensais (DNPM, 2005), respondendo por cerca de $25 \%$ da produção areeira do Estado de São Paulo. O vale do Paraíba fornece areia principalmente para atender à demanda da região metropolitana de São Paulo, que consome $80 \%$ de sua produção. Essa atividade mineradora se apresenta de forma ostensiva no Vale do Paraíba e pode comprometer o uso futuro das águas e das terras agricultáveis que cedem crescente espaço para as cavas. Esse comporta- mento do setor requer atenção quanto ao ordenamento, planejamento e aprimoramento tecnológico da atividade de mineração (Bitar, 2000). Entretanto a extração de areia ocupa lugar de destaque entre as atividades econômicas da região. A redução dessa atividade, segundo Texier (2001), não é cogitada em nível mundial, por se constituir no principal insumo da construção civil, ainda sem um sucedâneo à altura.

O consumo de areia no Brasil é da ordem de 1,8 toneladas/habitante/ano. Segundo Texier (2001), a França tem uma superfície de $547.030 \mathrm{~km}^{2}, 60$ milhões de habitantes, produz e consome 6,5 toneladas/habitante/ano.

Nesse contexto, o presente trabalho foi elaborado com o propósito de investigar as consequiências da extração de areia em larga escala no balanço hídrico climatológico do Vale do Paraíba, no trecho paulista, e relacionou o uso consuntivo de água dessa atividade e seu impacto nos diversos usos conflitivos, como o abastecimento urbano e o cultivo de arroz irrigado que é uma atividade tradicional na região.

\section{Materiais e métodos}

Aárea de estudo localiza-se no Vale do Paraíba paulista, entre as duas principais cidades do país, São Paulo e Rio de Janeiro, ligadas, desde 1950, pela mais importante estrada do Brasil: a Rodovia Presidente Dutra.

O mapeamento da área ocupada pelas cavas de areia e análise de sua evolução na área de estudo foram realizados utilizando-se o software SPRING - Sistema de Processamento de Informações Georreferenciadas (Câmara et al., 1996). Imagens orbitais correspondentes às cenas 218/76 e 219/76 do sensor TM do satélite Landsat 5 de dezembro de 1993 e agosto de 1997; e do sensor $\mathrm{ETM}^{+}$do Landsat 7, de fevereiro de 2003, e dados dos mapas de Zoneamento Minerário do Vale do Paraíba IG/SMA (Nogueira et al., 2001), escala 1:25.000, constituíram a base desse estudo. Esses dados foram integrados em um banco de dados geor- referenciado, implementado na plataforma SPRING, e permitiram a obtenção de mapas das ocorrências de espelhos d'água associados às cavas de extração de areia. Operações aritméticas diversas executadas no SPRING permitiram a quantificação da área ocupada pela atividade mineradora de areia no trecho entre os municípios de Jacareí e Pindamonhangaba, no médio Vale do Paraíba Paulista, no período de 1993 a 2003.

Para se gerar o balanço hídrico da região de estudo, realizou-se um levantamento dos dados hidrológicos e climatológicos necessários para o cálculo da estimativa da evaporação de lagos, a partir de uma série temporal de 11 anos (1993 a 2003). Sleight (1917) observou que a evaporação num tanque de $20 \mathrm{~m}^{2}$ não diferiu, significativamente, daquela obtida num lago de 6,9 ha. Esse autor propõe que, para se estimar a evaporação de um lago, a evaporação observada em um tanque com diâmetro menor que 3,66 m (situação-padrão dos postos meteorológicos) deve ser corrigida por um fator conhecido como coeficiente de tanque (Kp), que reduz a ECA (Evaporação em Tanques Classe A), ao valor aproximado da evaporação do lago (EL).

$\mathrm{EL}=\mathrm{Kp} . \mathrm{ECA}$

Segundo Pereira et al. (1997), em virtude da facilidade de se medir a evaporação utilizando tanques, esta tem sido a metodologia mais utilizada para se estimar a evaporação de lagos. Esse autor sugere um valor fixo igual a 0,66 para o Kp, que, freqüentemente, é arredondado para 0,7. Conceição (2002) estimou os valores da evapotranspiração de referência (ETo), para as condições da região noroeste do Estado de São Paulo, a partir de dados do tanque Classe A (ECA), utilizando diferentes métodos de determinação do coeficiente do tanque (Kp). O valor médio anual de Kp, determinado a partir da relação entre ECA e ETo, determinada pelo método PenmanMonteith-FAO (EToPM), foi de 0,74. Oliveira (1971), tomando o tanque de $20 \mathrm{~m}^{2}$ como padrão, nas condições de clima tropical, em Piracicaba, obteve coeficientes de tanque Classe A de 0,67 a 0,89, 
com média de 0,76 . Nesse trabalho, utilizou-se o método do Tanque Classe A, para estimar a evaporação dos lagos produzidos pelas cavas de areia e adotouse o valor fixo de $\mathrm{Kp}=0,76$, por se considerar ser o que melhor representa a região, com base nos resultados de Oliveira(1971).

Os dados climatológicos para o cálculo do Balanço Hídrico Climatológico e da estimativa da Evaporação de Lagos (EL) foram obtidos no Posto Meteorológico do Departamento de Ciências Agrárias da UNITAU, estação 83784 - UNITAU / INMET, Latitude $23^{\circ} 02^{\prime}$ S, Longitude $45^{\circ} 31^{\prime} \mathrm{W}$ e altitude $577 \mathrm{~m}$.

Apesar de a bacia do Rio Paraíba do Sul ser uma das mais estudadas no país, são poucos os dados hidrológicos e climatológicos disponíveis para a área de várzea. As informações disponíveis no Sistema Integrado de Gerenciamento de Recursos Hídricos, SIGRH (2005), em sua maioria, não oferecem uma série temporal completa. Foram selecionadas as estações E2-031, em Jacareí, E2-032, E2036 e E2-099, em São José dos Campos; E2-001 e E2-048, em Caçapava; E2-014, E2-041, E2-070 e E2-072, em Pindamonhangaba, localizadas em altitudes próximas à altitude da estação 83784 - UNITAU / INMET. Foram analisados os dados disponíveis entre 1993 e 2003 dessas estações pluviométricas, para a estimativa de uma média de precipitação anual da área de estudo, para validação da utilização dos dados da estação da UNITAU, que possui uma série completa de dados para o período estudado.

A comparação das informações de precipitação obtidas da estação UNITAU/INMET com a precipitação média obtida das estações do DAEE localizadas às margens do rio Paraíba não evidenciou diferenças significativas, o que credencia a utilização dos dados da estação 83784 - UNITAU/INMET para o estudo em questão, visto que essa estação tem dados para todo o período analisado (Reis, 2005).

As informações foram processadas em uma planilha para obtenção das estimativas de Evaporação de Lagos (EL) provocada pelas cavas de areia no trecho estudado. Os valores das áreas referentes aos anos intermediários para compor uma série temporal de 11 anos (1993 a 2003) foram estimados por interpolação. Os dados climatológicos e o respectivo balanço hídrico foram calculados com base no método de Tornthwaite e Matter (1955) com a estimativa da evapotranspiração potencial baseada na evaporação de Tanque Classe A, com a utilização da planilha adaptada de D’Angiolella e Vasconcellos (2001).

\section{Resultados e discussão}

Uma análise realizada pelo INPE (2002) apontava a existência de 225 cavas de areia no trecho em estudo. Os resultados do presente trabalho, efetuados com base em imagens do sensor TM do Landsat-5 e do sensor ETM $^{+}$do Landsat-7, demonstraram um acentuado crescimento do número de cavas (127\%) no período de 1993 a 2003, (Tabela 1).

O monitoramento da evolução da atividade minerária com o uso de imagens de satélite e sistemas de informações georreferenciadas demonstra um notável crescimento na área ocupada pelas cavas de areia durante o período estudado. De 1993 a 2003, a área de lagos artificiais originados pela extração de areia evoluiu de 591,4 ha para $1.726,5$ ha (Tabela 2). Isso corresponde a um crescimento percentual de $192 \%$. A visualização espacializada da evolução da área ocupada pela atividade de extração de areia em cava mostra o avanço da atividade (Comparação das Figuras 1 e 2).

Utilizando-se as áreas de cavas da Tabela 2 e as áreas estimadas por interpolação para os anos 1994, 1995, 1996, 1998, 1999, 2000 e 2001, assim como as informações sobre a precipitação anual e evaporação Tanque Classe A obtidas com os dados da estação 83784 (UNITAU/INMET) e assumindose um $\mathrm{Kp}=0$, 76, obteve-se o volume d'água perdido para a atmosfera por evaporação de lagos (Tabela 3).

Comparando a perda de água, por evaporação das áreas de cavas, com o uso da água para arroz irrigado, observa-se que este apresenta uma evaporação da ordem de $40 \mathrm{~m}^{3} / \mathrm{ha} /$ dia para a região do Vale do Paraíba SP(Telles, 1986), por aproximadamente cem dias por ano, o que corresponde a um total de $4.000 \mathrm{~m}^{3} / \mathrm{ha} /$ ano. Machado et al. (2006) reportam valores ainda mais altos $\left(75 \mathrm{~m}^{3} / \mathrm{ha} / \mathrm{dia}\right)$ para o ciclo do arroz irrigado na região do Rio Grande do Sul, dependendo do sistema de plantio. $\mathrm{Na}$ Tabela 3, pode ser observado que, no ano de 2003, houve um total de 19.157.022 $\mathrm{m}^{3} /$ ano evaporados por uma área total de cavas de 1.726,5 ha, o que representa um volume de evaporação de $11.099,1 \mathrm{~m}^{3} / \mathrm{ha} / \mathrm{ano}$. Dessa forma, em ter-

Tabela 1 - Cavas de areia entre Jacareí e Pindamonhangaba.

\begin{tabular}{ccccc}
\hline Ano & 1993 & 1997 & $2002 *$ & 2003 \\
\hline$N^{\circ}$ de Cavas & 113 & 178 & 225 & 256 \\
\hline${ }^{*}$ INPE (2002). & & &
\end{tabular}

Tabela 2 - Evolução da área (ha) minerada entre 1993 e 2003.

\begin{tabular}{lcccc}
\hline Área de estudo & 1993 & 1997 & $\mathbf{2 0 0 2}\left({ }^{*}\right)$ & $\mathbf{2 0 0 3}$ \\
\hline $\begin{array}{c}\text { Trecho entre Jacareí e } \\
\text { Pindamonhangaba }\end{array}$ & 591,4 & $1.022,80$ & $1.537,50$ & $1.726,50$ \\
\hline *INPE (2002). & & & & \\
\hline
\end{tabular}




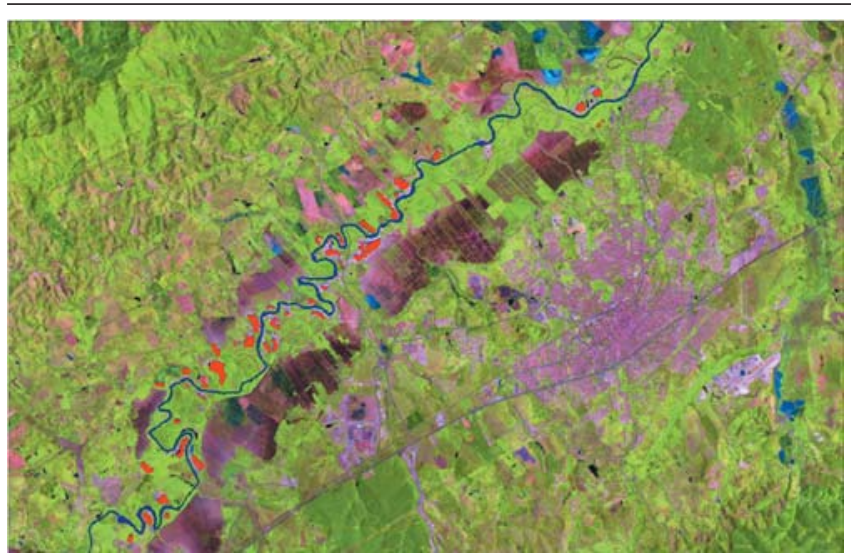

Figura 1 - Cavas de areia - ano 1993 (em Vermelho).

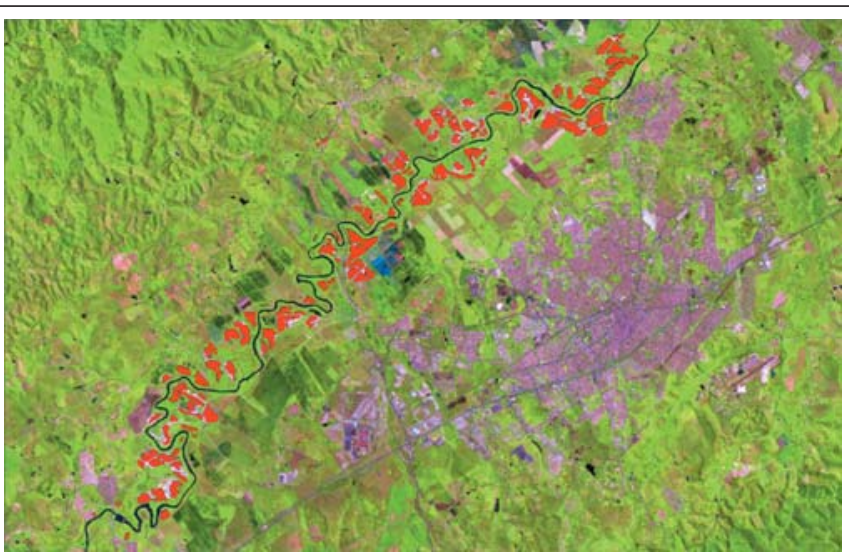

Figura 2 - Cavas de areia - ano 2003 (em Vermelho).

Tabela 3 - Estimativa das perdas de água para a atmosfera por evaporação em áreas de lagos das cavas de extração de areia.

\begin{tabular}{|c|c|c|c|c|c|c|c|}
\hline Ano & $\begin{array}{c}\text { Área lagos } \\
\text { (ha) }\end{array}$ & $\begin{array}{c}\text { Prec. anual } \\
\text { (mm/ano) }\end{array}$ & $\begin{array}{c}\text { Prec. na área } \\
\text { de lagos } \\
\left(\mathrm{m}^{3} / \mathrm{ano}\right)\end{array}$ & $\begin{array}{c}\text { ECA } \\
\text { (mm/ano) }\end{array}$ & $\begin{array}{c}\mathrm{EL}=\mathrm{Kp} \\
\mathrm{ECA} \\
\text { (mm/ano) }\end{array}$ & $\mathrm{EL}\left(\mathrm{m}^{3} / \mathrm{ano}\right)$ & \% EL/Prec. \\
\hline 1993 & 591 & 1.432 & 8.468 .132 & 1.405 & 1.068 & 6.313 .536 & 75 \\
\hline 1994 & 699 & 1.220 & 8.532 .490 & 1.452 & 1.103 & 7.714.356 & 90 \\
\hline 1995 & 807 & 1.493 & 12.048 .016 & 1.317 & 1.001 & 8.078 .175 & 67 \\
\hline 1996 & 915 & 1.608 & 14.713 .110 & 1.320 & 1.003 & 9.177 .958 & 62 \\
\hline 1997 & 1.023 & 1.086 & 11.103 .517 & 1.403 & 1.066 & 10.906.689 & 98 \\
\hline 1998 & 1.126 & 1.253 & 14.099 .818 & 1.341 & 1.019 & 11.473 .031 & 81 \\
\hline 1999 & 1.229 & 1.316 & 16.174 .186 & 1.420 & 1.079 & 13.256 .984 & 82 \\
\hline 2000 & 1.332 & 1.552 & 20.670 .458 & 1.353 & 1.028 & 13.692 .597 & 66 \\
\hline 2001 & 1.435 & 1.146 & 16.444 .086 & 1.523 & 1.158 & 16.608 .828 & 101 \\
\hline 2002 & 1.537 & 1.365 & 20.991 .078 & 1.511 & 1.149 & 17.659 .196 & 84 \\
\hline 2003 & 1.726 & 1.033 & 17.841 .444 & 1.460 & 1.110 & 19.157 .022 & 107 \\
\hline \multicolumn{2}{|c|}{ Totais } & 14.505 & 161.086 .335 & 15.505 & 11.784 & 134.038 .372 & 83 \\
\hline
\end{tabular}

mos anuais, as cavas de areia consomem, aproximadamente, 2,8 vezes mais água do que o cultivo do arroz irrigado por unidade de área. Portanto a água perdida por evaporação das cavas de areia seria suficiente para o plantio de 4.789 ha de arroz irrigado no Vale do Paraíba. Esse resultado levanta a reflexão sobre o censo conservador de que a agricultura irrigada é a maior consumidora de água conforme afirma Christofidis (1999), pelo menos localmente.
Para o ano de 2003, pior ano hidrológico dos últimos 50 anos (SIGRH, 2005), a perda de água para a atmosfera pela evaporação das cavas foi de 19.157.022 m³/ano (Tabela 3), o que corresponde a 52.484.991,8 1/dia. Considerando a média de consumo per capita do Estado de São Paulo de 160,84 1/hab/dia (SNIS, 2001), a perda de água para a atmosfera em 2003 seria suficiente para abastecer uma cidade com 326.318 habitantes.
Em termos climatológicos, o ano de 2003 foi o mais crítico, considerando-se que a precipitação total anual (1033 mm) e a média anual $(1.319 \mathrm{~mm})$, no período estudado, ficaram abaixo do valor médio (1.347,2 $\mathrm{mm})$ de 30 anos determinado por Lemos (2000), o que resultou no menor índice de reposição hídrica do balanço hídrico climatológico em todo o período (Figura 3). 
O balanço hídrico para o ano de 2003 demonstrou que esse foi um ano crítico, em que o déficit hídrico total anual $(249,1 \mathrm{~mm})$ foi maior do que o excedente hídrico anual de 184,0 mm, o qual ocorreu apenas em dois meses (janeiro com 150,2 mm e novembro com $33,8 \mathrm{~mm})$. O maior déficit ocorreu em setembro $(63,8 \mathrm{~mm})$. Isso está consistente com resultados de Fisch (1995). Nesse ano de 2003, o total precipitado foi de $1033,4 \mathrm{~mm}$ e a evapotranspiração potencial de $1098,5 \mathrm{~mm}$, portanto uma diferença de $65,1 \mathrm{~mm}$. A evapotranspiração real foi de 849,4 mm.

\section{Conclusões}

A análise das imagens de satélite indicou um crescimento da área das cavas de $192 \%$ no período analisado (1993 a 2003), enquanto a evaporação da lâmina d'água formada pela extração de areia em cava apresentou um crescimento na evaporação de $203 \%$, no mesmo período. Essa diferença de $11 \%$ na proporção do crescimento entre a área das cavas e taxa de evaporação deve-se às variações climáticas ocorridas no período.

O crescimento observado na atividade de mineração de areia em cava, de $192 \%$ no período analisado (1993 a 2003) tende a aumentar visto que não existe substituto viável para esse minério na construção civil e o Brasil possui um consumo per capta de cerca de $1,8 \mathrm{~m}^{3} /$ habitante/ano, que é muito baixo, considerando-se a média européia que é de $6,8 \mathrm{~m}^{3} /$ habitante/ano.

As informações geradas, a partir do banco de dados georreferenciados, permitiram, ainda, mostrar, a partir da sobreposição dos dados do zoneamento minerário, que o crescimento das áreas de extração de areia tem ocorrido, também, nas áreas de restrição do Zoneamento Ambiental Minerário, zonas de Preservação, Recuperação e Conservação de Várzea.

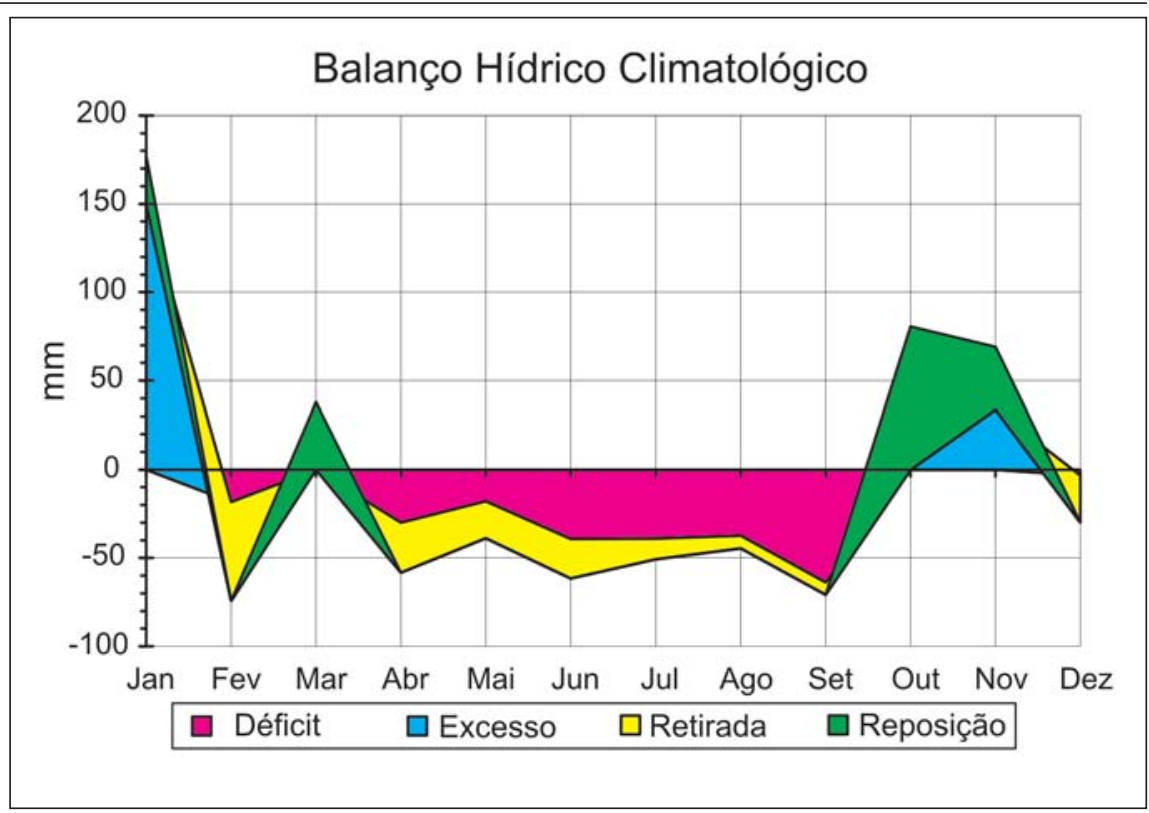

Figura 3 - Balanço hídrico climatológico para o ano de 2003.

\section{Referências bibliográficas}

BITAR, OMAR YAZBEK, IYOMASA, WILSON SHOJI, CABRAL JR., MARSIS. Geotecnologia: tendências e desafios. São Paulo Perspec., v.14, n.3, p.78-90, July/Sept. 2000, ISSN 01028839.

CÂMARA, G., SOUZA, R.C., FREITAS, U. SPRING integrating remote sensing and GIS with object oriented data modelling. Computers and Graphics, Dordrecht, Holanda. v. 15, n. 6, p. 1322, 1996.

CHRISTOFIDIS, D. Recursos Hídricos e Irrigação no Brasil. Brasília: CDC/UnB, 1999. 34p.

CONCEIÇÃO, M. A. F. Reference evapotranspiration based on class A pan evaporation. Scientia Agricola, v.59, n.3, p.417-420, July/Sept. 2002, ISSN 01039016.

D’ANGIOLELLA, G., VASCONCELLOS, V. L. Planilhas excel para cálculo de balanço hídrico climatológico com diferentes metodologias para estimativa da evapotranspiração potencial. In: ENCUENTRO DE LAS ÁGUAS, 3. Anais... Santiago-Chile, 24 a 26 Out. 2001.

DNPM - DEPARTAMENTO NACIONAL DA PRODUÇÃO MINERAL. Anuário mineral brasileiro. Brasília: Departamento Nacional da Produção Mineral, 2005. v. 34, 426p.
FISCH, G. Caracterização climática e balanço hídrico de Taubaté, São Paulo. Revista Biociências, v.1, n.1, p.81-90, 1995.

INPE - Instituto Nacional de Pesquisas Espaciais - Coordenação Geral de Observação da Terra (OBT-DSR). Análise temporal da mineração na Várzea do Rio Paraíba do Sul -SP no período entre os anos de 1986 e 2002. 2002.

LEMOS C. F. Caracterização e variabilidade climática do vale do Paraíba. Universidade de Taubaté: Programa de Pós-Graduação em Ciências Ambientais, 2000. (Dissertação de Mestrado).

MACHADO, Sérgio Luiz de Oliveira, MARCHEZAN, Enio, RIGHES, Afrânio Almir et al. Water use and nutrients and sediments losses on the initial water drainage on flooded rice. Cienc. Rural. [online], v. 36, n. 1, p. 65-71, 2006. < http://www.scielo.br/

NOGUEIRA, S.A.A., TEIXEIRA, A.L., MONTANHEIRO, T.J., SHIMADA, H., NEGRI, F.A. O zoneamento ambiental minerário da extração de areia no vale do Rio Paraíba do Sul, São Paulo, Brasil. In: CONGRESSO LATINOAMERICANO DE GEOLOGIA, 11. Actas... Montevidéu, 2001. Montevideo, DINAMIGE/SUG, Trabalho n.158, 4p. (Edição eletrônica).

OLIVEIRA, A. S. Estudos comparativos da evaporação potencial estimada por tanques e pelo método de Penman. ESALQ/USP, 1971. 113p. (Tese de Livre Docência). 
PEREIRA, A. R., VILA NOVA N. A, SEDIYAMA, G. C. Evapo(transpi)ração. Piracicaba: FEALQ, 1997. 183p.

REIS, B. J. Avaliação da expansão da atividade de extração de areia na planície aluvial da bacia hidrográfica do rio Paraíba do Sul no trecho entre Jacareí e Pindamonhangaba no período de 1993 a 2003. Universidade de Taubaté: 2005. 112 p. (Dissertação de Mestrado em Ciências Ambientais).

SÃO PAULO (Estado). Secretaria de Obras e do Meio Ambiente. Departamento de Águas e Energia Elétrica Estudo de Águas Subterrâneas, 1977.

SÃO PAULO (Estado). Secretaria de Recursos Hídricos Saneamento e Obras. Documento Estratégico de Negociação -
Projeto de Qualidade das Águas e Controle da Poluição na bacia do rio Paraíba do Sul, 1999.

SIGRH - Sistema Integrado de Gerenciamento de Recursos Hídricos do Estado de São Paulo, Base Georreferencial; Pluviométrico.http://www.sigrh.sp.gov.br/ cgi-bin/bdhm. Acesso em 15 de março de 2005.

SLEIGHT, R.B. Evaporation front of surfaces of water and river-bed materials. Journal of Agricultural Resources, 10, p. 209-262, 1917.

SNISS - Sistema Nacional de Informações Sobre Saneamento. Diagnóstico dos serviços de água e esgotos - 2001. http:/ /www.snis.gov.br/\#. Acesso em 04/05/ 2006.
TELLES, D.A., Org. Drenagem agrícola. Ed. Associação Brasileira de Irrigação e Drenagem (ABID), EPUSP, DAEE. 1986. 285p.

TEXIER, G. Visão Governamental Européia. In: INTERNATIONAL SEMINAR ON CONSTRUCTION AGGREGATES. Anais... São Paulo: ANEPAC, 2001. p. 24-28.

THORNTHWAITE, C. W., MATHER, J. R. The water balance. Centerton, Drexel Institute of Technology-Laboratory of Climatology, Climatology, v. 8, n.1, 1955. $104 \mathrm{p}$.

VALVERDE, F. M. Agregados para a construção civil. Balanço Mineral Brasileiro. DNPM, 2001.

Artigo recebido em 08/05/2006 e aprovado em 08/10/2006.

\title{
Descubra as muitas informações da:
}

\author{
Geologia, Mineração, \\ Metalurgia \& Materiais \\ e Engenharia Civil.
}

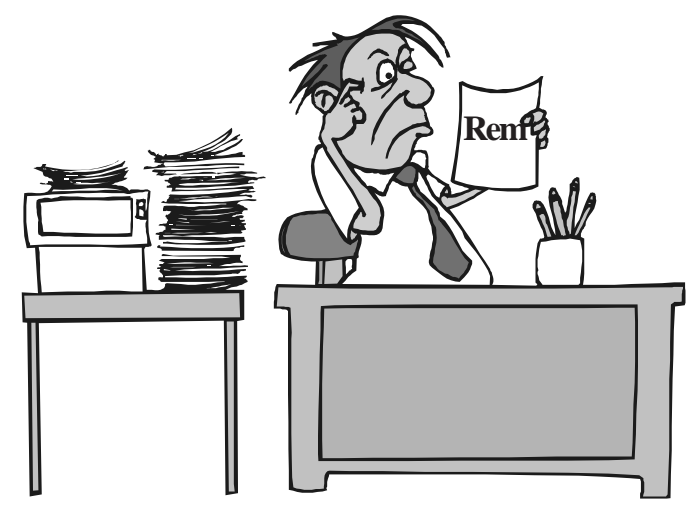

\section{Assine e publique na Rem}

Conheça o nosso novo site: WWW.rem.com.br 